\section{Comparative Evaluation of Continuous Infusion vs. Intermittent Doses of $0.2 \%$ Ropivacaine in Adductor Canal Block for Postoperative Analgesia in Total knee replacement Surgery: Randomized Double- Blind Study}

\section{Abstract}

Background: Total Knee Replacement (TKR) surgery is associated with moderate to severe pain post-operatively. Multimodal analgesic regimens have been the mainstay of pain therapy after TKR. ACB is a highly successful approach favouring reduced post-operative opioid requirements and facilitates in earlier ambulation.

Method: In this prospective randomized, double-blind, placebo-controlled, parallel group study, 110 ASA class I-II adult patients of either sex, scheduled for Unilateral Total Knee Arthroplasty(TKA) under spinal anesthesia, were included. Out of which 62 patients were recruited and randomly assigned to their treatment group. ACB was performed immediately postoperatively. Patients were randomized to receive nociception level-guided analgesia by either Ropivacaine or placebo based infusion in orthopaedic ICU. Infusion was given at $8 \mathrm{ml} / \mathrm{h}$, and rescue local analgesic was given if pain score of $\geq 3$, if not relieved then opioid supplementation was done.

Result: Both continuous and intermittent group showed no statistically significant results in terms of pain score $\geq 3(2 / 28 \mathrm{vs.} 5 / 28 p=0.21)$, rescue Ropivacaine boluses requirement $(5 / 28$ vs. $2 / 28 ; p=0.21)$ or morphine consumption $(0 / 28$ vs. $2 / 28$; $\mathrm{p}=0.491$ ) in $24 \mathrm{~h}$ postoperatively. We observed significant differences between the groups in outcomes like Ropivacaine consumption and motor blockade. At $16^{\text {th }} \mathrm{h}$, bromage score $\geq 1$ was $20 / 28$ vs. $2 / 28$ ( $p=0.000$ ) hence, limiting ambulation. But, at $24^{\text {th }} \mathrm{h}$, only 1 patient in the continuous group had a motor blockade. Ropivacaine consumption of $24 \mathrm{~h}$ was $(193.43 \pm 5.25 \mathrm{ml})$ vs. $(4.29 \pm 9.97 \mathrm{ml}),(p=0.000)$.

Conclusion: Intermittent ACB during TKA is favourable over continuous ACB in terms of pain, opioid requirement, and motor blockade.

Keywords: ACB; Knee surgery; Ropivacaine; TKA; Saphenous nerve block
Kavita Kadyan ${ }^{1}$, Padma Puppala ${ }^{2}$ and Dinesh Kumar Kataria ${ }^{3}$

1 Department of Anesthesiology, AlIMS, New Delhi, India

2 Department of Anaesthesiology and Critical Care, Fortis Hospital, Mohali, Punjab, India

3 Department of Anesthesia and Critical Care, Fortis Hospital, Mohali, Punjab, India

*Corresponding author: Kavita Kadyan

झ kavitakadyan.kk@gmail.com

Department of Anesthesiology, Pain Medicine and Critical Care, All India Institute of Medical Sciences, New Delhi, India.

Tel: 9915093161

Citation: Kadyan K, Puppala P, Kataria DK (2019) Comparative Evaluation of Continuous Infusion vs. Intermittent Doses of $0.2 \%$ Ropivacaine in Adductor Canal Block for Postoperative Analgesia in Total knee replacement Surgery: Randomized DoubleBlind Study. Int J Anesth Pain Med. Vol.5 No.1:2

Received: October 10, 2019; Accepted: October 30, 2019; Published: November 08, 2019

\section{Introduction}

TKR surgery is associated with moderate to severe pain postoperatively [1,2]. Most of these patients are elderly with significant co-morbidities, and inadequate pain relief can lead to discomfort, stress, and immobilization with secondary medical sequelae like venous thromboembolism, infections thus increasing morbidity, medical cost, and decreasing the postoperative quality of life [3].
Multimodal analgesic regimens including neuraxial analgesia or regional anaesthesia, with systemic analgesics, have been the mainstay of pain therapy after TKR [4].

However, due to their association with complications like, in case of parental opioid therapy with sedation and respiratory depression, in epidural analgesia with urinary retention and motor block [5], femoral nerve block with reduced quadriceps muscle strength, hematoma formation and permanent nerve 
injury [6-8] will lead to the emergence of ACB also known as Saphenous nerve block or Hunter's canal block (Saphenous nerve is the largest terminal sensory branch of the femoral nerve)as a highly successful approach $[9,10]$ having advantages of lesser decrease in quadriceps muscle strength [11] and lower pain scores on movement. $A C B$ also reduces post-operative opioid requirements and facilitates earlier ambulation $[12,13]$.

Administration of high-volume local anesthetic into the adductor canal has been shown to be a useful option for postoperative analgesia after TKA [14,15]. Postsurgical pain lasts for many days, whereas the action of local anesthetic lasts for 18 to $24 \mathrm{~h}$ after a single shot injection. Duration of action can be prolonged with additives such as epinephrine or a corticosteroid like a dexamethasone [16]. Alternatively, indwelling nerve block catheters can be placed adjacent to the nerve and a local anesthetic infused through an infusion pump [17]. We hypothesized that ACB would improve pain relief (primary endpoint), reduce morphine consumption and enhance ambulation ability (secondary endpoints) after TKA compared with placebo. Hence, this prospective, randomized, double-blind, placebo-controlled study was conducted to evaluate the efficacy of $A C B$ groups on Ropivacaine and Opioid consumption, pain relief and ambulation ability after TKA.

\section{Methods}

\section{Study design}

The study had a double-blinded, randomized, parallel group design and was performed in a single tertiary centre. Patients were randomized to receive continuous or intermittent infusion of ropivacaine using a computer-generated randomization list.

All investigators, staff, and patients were blinded to the treatment groups. The randomization key was first broken once enrolment of all patients was completed and data computed. Patients were not informed on their group assignment and could not discern to what group they were allocated.

\section{Study place}

Orthopedic Operation Theatre and Orthopedic Intensive Care Unit facility of a tertiary care centre, Fortis Hospital, Mohali.

\section{Study period}

1 year, March 2017-March 2018

\section{Patient selection}

ASA class I-II patients (aged 45 to $75 \mathrm{yr}$ ) of either sex, scheduled for elective unilateral TKA under spinal anesthesia, were recruited to participate in the study.

\section{Exclusion criteria}

Inability to give informed consent, patient's refusal, age $<45$ years and $>75$ years, belonging to ASA grade III-IV, obese patients with body mass index $>35 \mathrm{~kg} / \mathrm{m}^{2}$, patients with obstructive sleep apnoea syndrome, allergy to any of the study medications/local anesthetic agent, Patient with substance abuse, chronic opioid use, having hearing or cognitive dysfunctions, inflammation or infection at the injection site, on anticoagulation drugs or bleeding disorders, pre-existing peripheral neuropathies, patients with failed spinal anesthesia, long standing diabetes $>20$ years and with residual CVA/Central nervous system disorder.

\section{Procedure}

The patients were kept nil per oral for $8 \mathrm{~h}$ before the surgery and a premedication of tablet Alprazolam $0.25 \mathrm{mg}$ and tablet Ranitidine $150 \mathrm{mg}$ were given $2 \mathrm{~h}$ prior to surgery according to institutional protocol. Upon arrival in the operating room, the patients received an intravenous access line and were connected to standard monitors (3-lead continuous electrocardiogram, non-invasive blood pressure by arm cuff, pulse oximetry by finger probe).

Subarachnoid block was induced with 26 G Quincke needle, with $2.5-3.0 \mathrm{ml}$ of $0.5 \%$ hyperbaric bupivacaine at the L3-4 or L2-3 vertebral interspaces. The ACB was performed immediately postoperatively. Structures were identified by using highfrequency $(5-12 \mathrm{~Hz})$ linear ultrasound transducer in transverse plane. The sartorius, which is lateral to the artery in upper onethird of the thigh, crosses the artery in mid-thigh, end traverses to the medial side of the artery in lower one-third of thigh. Most favourable site for ultrasound probe placement in our study was the medial aspect of mid-thigh where saphenous artery lies just posterior to sartorius muscle. Underneath the sartorius muscle the femoral artery was identified, with the vein just inferior and the saphenous nerve just lateral to the artery.

An $18 \mathrm{G}, 11 \mathrm{~cm}$ long, Tuohy's needle was inserted $2-3 \mathrm{~cm}$ lateral to the probe, directing the needle medially and toward a point slightly lateral to the artery, using "in-plane" technique. Hydrodissection with 0.5 to $1 \mathrm{ml}$ aliquots of saline is used until the desired plane is reached. Space was confirmed when the injected saline spreads in an inverted " $U$ " shaped or lenticular shape or horse shoe pattern beneath the inferior aponeurosis of sartorius muscle, and the spread is anterior or anterolateral to the artery (Figure 1). After aspirating, both the groups were given $0.75 \%$ Ropivacaine $30 \mathrm{ml}$ and $4 \mathrm{mg}$ dexamethasone was given in adductor canal. Once the spread is satisfactory, $21 \mathrm{G}$ catheter was then inserted $10-15 \mathrm{~cm}$ through the Tuohy's needle, based on depth of the tuohy's needle to ensure a minimum of $5 \mathrm{~cm}$ length of catheter in nerve space and further $5 \mathrm{ml}$ of study medication under US guidance was given through it.

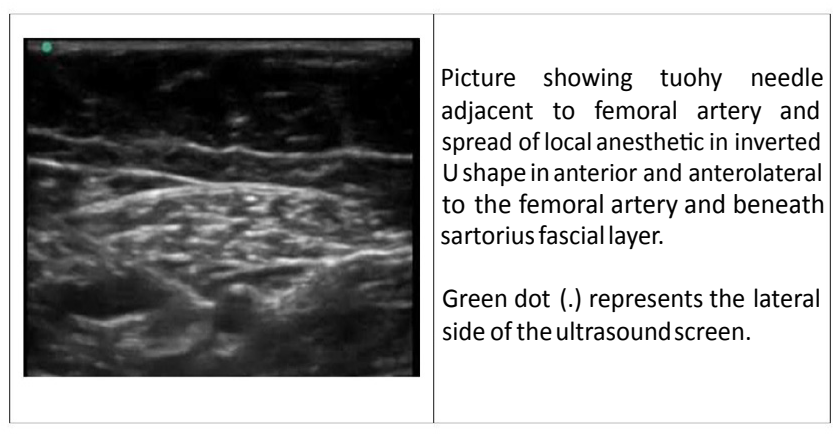

Figure 1 Ultrasonographic view of Adductor canal block. 


\section{Postoperative management}

After the surgery the patient was moved to the orthopedic intensive care unit (ICU). Patients were randomly assigned to receive either ACB with ropivacaine $0.2 \%$ or $0.9 \%$ isotonic saline at $8 \mathrm{ml} / \mathrm{h}$.

Additional boluses of $20 \mathrm{ml}$ of $0.2 \%$ ropivacaine was administered when patient reported pain score of $\geq 3$ in both the groups. If no analgesic response was obtained in 15 mins then rescue opioid, Morphine $0.05 \mathrm{mg} / \mathrm{kg}$ IBW (ideal body weight) was given intravenously.

Additional analgesics: Inj. Paracetamol 1 gm IV 6 hrly and Inj. Diclofenac 75 mg IV 12 hrly.

Thromboprophylaxis: Inj. Enoxaparin $1 \mathrm{mg} / \mathrm{kg}$ once daily for 2 days.

Ulcer prophylaxis: Tab. Pantaprazole $40 \mathrm{mg}$ once daily.

Rescue anti-emetic: Inj. Ondansetron $8 \mathrm{mg}$ IV as and when required.

\section{Data collection}

Data were derived from three parameters obtained every $4^{\text {th }}$ hrly till $24 \mathrm{~h}$.

(1) Motor blockade scale, Bromage Score [18]

(2) Pain assessment scale, NPRS score $[19,20]$

(3) And the electronic medical record database (Fortis Hospital, Mohali): MAP, heart rate.

\section{Ethical approval}

The Ethical Committee Board of Fortis Hospital Mohali, Punjab approved the protocol. The patients were recruited from March 2017 to March 2018. There were no amendments to the study protocol. All patients received written information about the protocol, had ample time to decide on their participation, and gave oral and written informed consent before enrolment into the study

\section{Statistical analysis}

The statistical analysis of the data was carried out using Statistical Package for Social Sciences (SPSS Inc., Chicago, IL, version 17.0 for Windows). All quantitative variables e.g. PR, MAP were estimated using mean, and standard deviation; Scores or skewed data were presented as median. Normality of data was checked by measures Kolmogorov Smirnov tests of normality. For normally distributed data means of quantitative variables of two groups were compared using student t-test. For skewed data or scores, Mann Whitney test was applied. Qualitative or categorical variables were described as frequencies and proportions. Proportions were compared using Chi-square or Fisher's exact test whichever was applicable. For time-related variables (quantitative data) Repeated Measure ANOVA was applied. For time-related scores, Wilcoxon Signed Rank test was applied. All statistical tests were two-sided and were performed at a significance level of $\alpha=0.05$. The analyses were restricted to data related to the primary and secondary endpoints. Data are presented as mean \pm SD, P values less than 0.05 were considered significant.

\section{Sample size}

The sample size was estimated based on the following 2 studies. A randomized study by Grevstad [21]: Effect of ACB on pain in patients with severe pain after total knee arthroplasty and a randomized controlled trial by Shah Adductor Canal Blockade Following Total Knee Arthroplasty Continuous or Single Shot Technique role in postoperative: analgesia, ambulation ability, and early functional recovery. Our sample size came out to be 28 subjects per group at a power of $80 \%$ and a confidence interval of $95 \%$. We had taken 31 subjects in each group for any exclusion [22].

\section{Results}

A total number of 110 patients were approached for participation in the study. Out of which 62 patients were recruited and randomly assigned to their treatment group, of these 6 patients were excluded after randomization (Figure 2). Finally, data from 56 patients were analyzed.

Surgical procedure and anesthesia times were similar between treatment groups (data not shown).

All study patients completed the trial without any harms or adverse events, and there we no missing data.

The groups were similar with respect to demographics and perioperative data (Table 1).

NRPS pain scores from 0 to $24 \mathrm{~h}$ post-operatively were

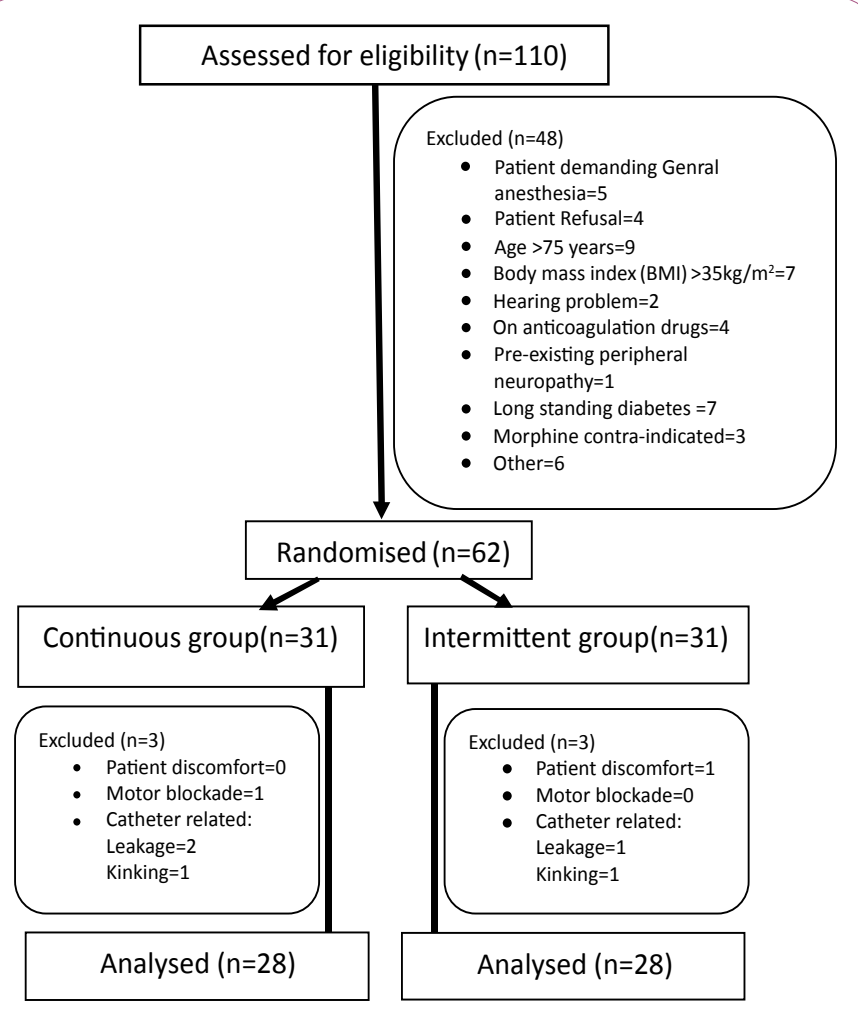

Figure 2 Screening of patients. 
comparable in both the groups, $2 / 28(7.1 \%)$ reported NRPS score $\geq 3$ in Group C and 5/28 (17.9\%) in Group I ( $p=0.21)$

Ropivacaine consumption of the $24 \mathrm{~h}$ was found to be statistically significant $(p=0.000)$. In Group $C$ and I, the mean ropivacaine requirement was $193.43 \pm 5.25$ and $4.29 \pm 9.97$ respectively.

\section{Ropivacaine bolus requirement}

The distribution of rescue bolus requirement between the two groups was insignificant, $\mathrm{p}=0.21$ with $7.1 \%(2 / 28)$ and $17.9 \%$ $(5 / 28)$ in group $C$ and I respectively.

\section{Opioid drug consumption}

2/28 (7.1\%) patients in Group I and 0/28 (0\%) patients in Group $\mathrm{C}$ received rescue opioid (morphine) as per the study algorithm. The result was insignificant; $p=0.491$.

\section{Motor blockade}

Significant result in $16^{\text {th }}$ and $20^{\text {th }} \mathrm{h}$ (Figure 3).

\section{Complications}

During our study we encountered catheter related complications; mobility, motor blockade problems and few were vascular insertion problems.

As we had catheter insertion in our study, 3 cases with the

Table 1 Demographic distribution of patients.

\begin{tabular}{|c|c|c|}
\hline Parameters & Continuous group & Intermittent Group \\
\hline Age $(\mathrm{yr})$ & $64.14 \pm 3.40$ & $64.75 \pm 2.99$ \\
\hline Weight $(\mathrm{kg})$ & $75.39 \pm 6.17$ & $77.04 \pm 4.04$ \\
\hline Height $(\mathrm{cm})$ & $158.36 \pm 7.53$ & $157.64 \pm 6.27$ \\
\hline Sex (male/female) & $12 / 16$ & $11 / 17$ \\
\hline ASA I/II & $15 / 13$ & $18 / 10$ \\
\hline No comorbidities & 15 & 18 \\
\hline Diabetes & 02 & 02 \\
\hline Hypertension & 06 & 05 \\
\hline Hypothyroid & 05 & 03 \\
\hline
\end{tabular}

inadvertent passage of the catheter into the femoral vein or adjacent muscle layers occurred despite using ultrasound guidance, for which either re-insertion of the catheter was performed intra-operatively or the case was excluded from the study. Also, leakage and kinking of catheter were seen in $3 / 62$ and $1 / 62$ respectively despite proper fixation of the catheter at mid-thigh. Out of 62 only one case reported mobility issues as discomfort with the presence of the catheter in mid-thigh asking for the removal of the same, despite explaining the analgesic benefits. Motor blockade after $24 \mathrm{~h}$ postoperatively was present in one of 62 cases, thus, hampering mobilisation.

\section{Discussion}

The above study showed the statistical difference in local anesthetic and rescue opioid requirement between intermittent 'as necessary boluses' (on SOS) and 'continuous infusion' of $0.2 \%$ ropivacaine through adductor canal, via a catheter with a midthigh, subsartorial approach, in patients undergoing TKA for obtaining adequate postoperative analgesia.

Our study results showed that $2 / 28$ vs. $5 / 28$ patients reported pain score $\geq 3$ in continuous and intermittent $A C B$ group respectively, which was statistically insignificant $(p=0.21)$. Neither the groups showed any statistically significant increase either in the requirement of rescue ropivacaine boluses $(5 / 28$ vs. $2 / 28$; $p=0.21)$ or in morphine consumption $(0 / 28$ vs. $2 / 28 ; p=0.491)$ in $24 \mathrm{~h}$ postoperatively. Nevertheless, we observed significant differences in several outcomes between the study groups like ropivacaine consumption and motor blockade.

While comparing the mean ropivacaine consumption of $24 \mathrm{~h}$ among the groups, statistically significant results $(p=0.000)$ were obtained with continuous ACB $(193.43 \pm 5.25 \mathrm{ml})$ vs. intermittent $A C B(4.29 \pm 9.97 \mathrm{ml})$. Vastus medialis is the only muscle which affects the motor power of the lower limb in this block, thus making ACB almost a pure sensory block. We used $0.75 \%$ ropivacaine in relatively large volumes to ensure even distribution throughout the adductor canal. Our study assessed the patient's motor function during $24 \mathrm{~h}$ using Bromage score.

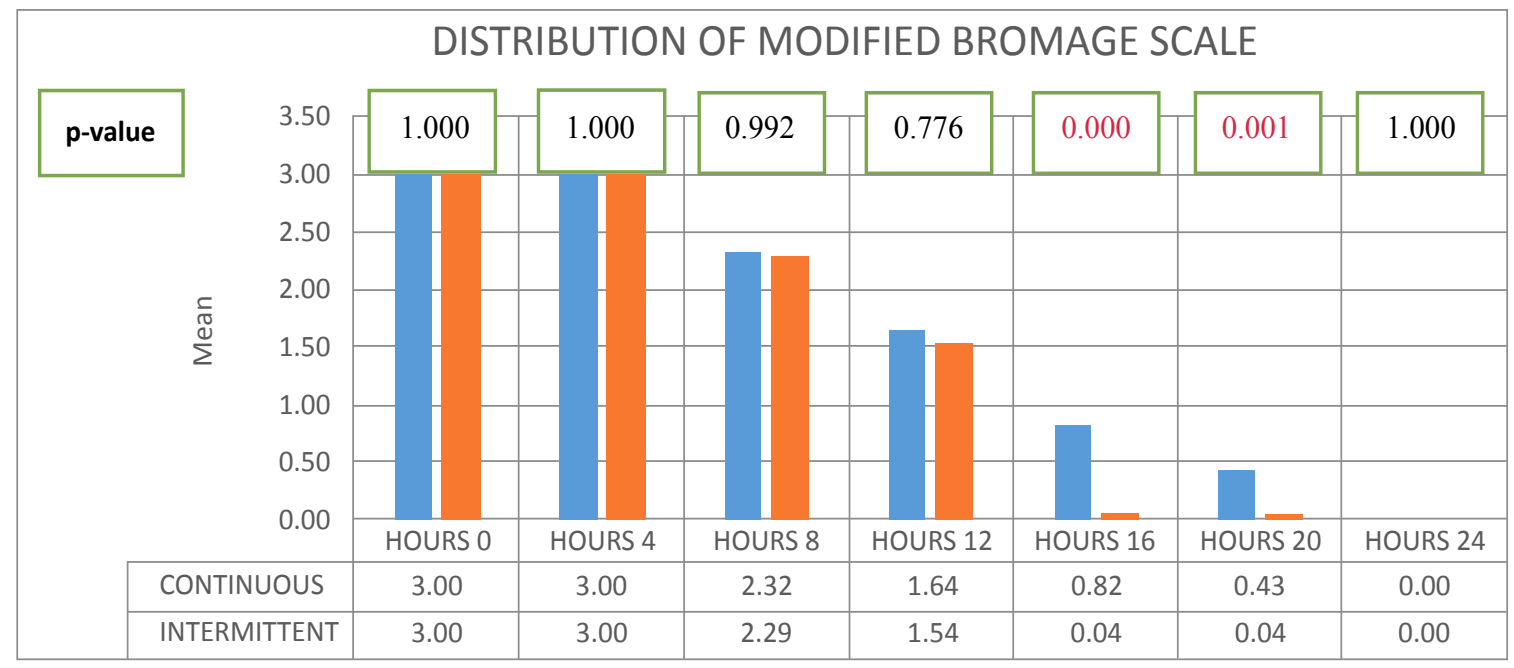


The results obtained show that the continuous infusion $A C B$ may limit early ambulation compared with intermittent infusion $A C B$. At $16^{\text {th }} \mathrm{h}, 20 / 28$ (continuous $A C B$ ) and $2 / 28$ (intermittent $A C B$ ) patients had a Bromage score of $\geq 1$ hence, limiting ambulation ( $p=0.000$ ). But, at $24^{\text {th }} h$, only 1 patient in the continuous group had a motor blockade and remaining patients in both the groups were ready for ambulation. Obviously, more studies must be done to establish the possible effect of $A C B$ on muscle strength.

The ACB is a novel technique and studies are needed to investigate the optimal concentration and volume of local anesthetic to be utilized in this block. US-guided ACB and adductor catheter insertion is a challenging and advanced technique needing a certain degree of skill to perform. In addition, indwelling catheters related complications can occur. In our study, $4.8 \%(3 / 62)$ patients had catheter leakage, $1.6 \%(1 / 62)$ kinking and $1.6 \%$ $(1 / 62)$ catheter removal. $4.8 \%$ (3/62) patients had an inadvertent passage of the catheter into the femoral vein or adjacent muscle layers, for which either re-insertion of the catheter was done intra-operatively or that case was excluded from the study.

There are controversies for obtaining the superior analgesia technique amongst continuous peripheral nerve blocks with a catheter technique and the single shot technique. Several studies showed a reduction in pain or morphine consumption during a continuous infusion compared with a control group [23-26] but only a few studies directly compare continuous infusion with a single-shot technique $[27,28]$.

Jaeger et al. examined the effect of ACB on established pain in the early postoperative period after TKA compared with placebo. They found no significant difference in VAS pain scores during active flexion of the knee at their primary endpoint $1 \mathrm{~h}$ after an operation or at rest, but a significant reduction in VAS during active flexion of the knee in favour of ACB [29].

Jenstrup et al. compared the effect of repeated ACB boluses, through a catheter, with those of placebo. They found a significant reduction in morphine consumption during the study period (0$24 \mathrm{~h}$ ), a significant reduction in pain scores during an active $45^{\circ}$ flexion of the knee, and a reduction in time used to perform a mobilization test [30].

Shah et al. compared the efficacy of continuous and single shot adductor canal block. They concluded that continuous adductor canal blockade is superior to single shot block in terms of pain control but was similar for early functional recovery.

\section{References}

1 Wylde V, Hewlett S, Learmonth ID, Dieppe $P$ (2011) Persistent pain after joint replacement: prevalence, sensory qualities, and postoperative determinants. PAIN 152: 566-572.

2 Guimaraes-Pereira L, Valdoleiros I, Reis P, Abelha F (2016) Evaluating Persistent Postoperative Pain in One Tertiary Hospital: Incidence, Quality of Life, Associated Factors, and Treatment. Anaesthesiology and pain medicine 6: e36461.

3 Carr DB, Goudas LC (1999) Acute pain. The Lancet 353: 2051-1058.
A study by Thapa et al. concluded that the intermittent ACB has significantly reduced consumption of morphine for $24 \mathrm{~h}$ in the post-operative period compared with continuous ACB when identical doses of ropivacaine were used in each group.

\section{Conclusion}

It can be concluded from the findings of present study that the intermittent group is comparable to continuous group in terms of pain score, motor blockade, and opioid dosage with the advantage of decreased dose of ropivacaine requirement. Hence, intermittent group can be favoured over continuous group in patients undergoing TKR surgery. Future studies should be done to address whether the analgesic effect demonstrated in the present study is comparable to that of single shot $A C B$ alone, and should evaluate the effect of $A C B$ on motor strength and mobilization in patients undergoing TKR. Further, optimal volumes, concentrations, and single shot vs. continuous infusion administrations should be studied in future.

\section{Contribution Details}

Padma Puppala: concept, design, definition of intellectual content, manuscript editing

Dinesh Kumar Kataria: manuscript preparation and manuscript review

Kavita Kadyan: definition of intellectual content, literature search, clinical studies, experimental studies, data acquisition, data analysis, statistical analysis, manuscript preparation, manuscript editing and manuscript review.

\section{Research Support}

This double blind trial was supported by institutional and departmental funds. Fortis Hospital Mohali, Punjab provided the hardware devices used in the study.

\section{Acknowledgments}

The authors thanks, Dr Adarsh Chander Swami, MD, Director, Department of Anaesthesiology and Critical Care, Fortis Hospital, Mohali, Punjab, India. He helped in conducting the cases and providing the facilities to perform the study. Dr Punit Khanna, $M D$, Consultant, Department of Anaesthesiology and Critical Care, AIIMS, New Delhi, India. He helped in reviewing the article for publication.

4 Mechel AE (2000) Ramsey: Acute postoperative pain management. Proc 13: 244-247.

5 Fowler SJ, Symons J, Sabato S, Myles PS (2008) Epidural analgesia compared with peripheral nerve blockade after major knee surgery: a systematic review and meta-analysis of randomized trials. $\mathrm{Br} \mathrm{J}$ Anaesth 100: 154-164.

6 Feibel RJ, Dervin GF, Kim PR, Beaulé PE (2009) Major complications associated with femoral nerve catheters for knee arthroplasty: a word of caution. J Arthroplasty 24: 132-137.

7 Charles MT, Madison SJ, Suresh PJ, Sandhu NS, Loland VJ, et al. (2011) 
Continuous Femoral Nerve BlocksVarying Local Anesthetic Delivery Method (Bolus versus basal) to Minimize Quadriceps Motor Block while Maintaining Sensory Block. American Society of Anesthetists 115: 774-781.

8 Ilfeld BM, Duke KB, Donohue MC (2010) The association between lower extremity continuous peripheral nerve blocks and patient falls after knee and hip arthroplasty. Anaesthesia and analgesia 111: 1552.

9 Van der Wal M, Lang SA, Yip RW (1993) Transsartorial approach for saphenous nerve block. Can J Anaesth 40: 542-546.

10 Mansour NY (1992) Sub-sartorial saphenous nerve block with the aid of nerve stimulator. Regional anaesthesia 18: 266-268.

11 Jaeger P, Zaric D, Fomsgaard JS, Hilsted KL, Bjerregaard J, et al. (2013) Adductor canal block versus femoral nerve block for analgesia after total knee arthroplasty: a randomized, double-blind study. Regional anesthesia and pain medicine. 38: 526-532.

12 Moore DM, O'Gara A, Duggan M (2013) Continuous saphenous nerve block for total knee arthroplasty: when and how? Regional Anaesthesia and Pain Medicine 38: 370-371.

13 Andersen HL, Gyrn J, Møller L, Christensen B, Zaric D (2013) Continuous saphenous nerve block as a supplement to single-dose local infiltration analgesia for postoperative pain management after total knee arthroplasty. Regional anaesthesia and pain medicine 38 : 106-111.

14 Jin SQ, Ding XB, Tong Y, Ren H, Chen ZX, et al. (2015) Effect of saphenous nerve block for postoperative pain on knee surgery: a meta-analysis. Int J Clin Exp Med 8: 368.

15 Bonet A, Koo M, Sabaté A, Otero I, Bocos J, et al. (2015) Ultrasoundguided saphenous nerve block is an effective technique for perioperative analgesia in ambulatory arthroscopic surgery of the internal knee compartment. Revista Española de Anestesiología y Reanimación 62: 428-435.

16 Rasmussen SB, Saied NN, Bowens C, Mercaldo ND, Schildcrout JS, et al. (2013) Duration of upper and lower extremity peripheral nerve blockade is prolonged with dexamethasone when added to ropivacaine: a retrospective database analysis. Pain Med 14: 12391247.

17 Karnawat R, Gupta M, Suthar OP (2015) Adductor Canal Block for Post-Operative Pain Relief in Knee Surgeries: A Review Article. J Anesth Clin Res 12: 2013.

18 Ousley R, Egan C, Dowling K, Cyna AM (2012) Assessment of block height for satisfactory spinal anaesthesia for caesarean section. Anaesthesia 67: 1356-1363.

19 Rodriguez CS (2001) Pain measurement in the elderly: a review. Pain Management Nursing 2: 38-46.
20 Jensen MP, McFarland CA (1993) Increasing the reliability and validity of pain intensity measurement in chronic pain patients. Pain 55: 195-203.

21 Jaeger P, Grevstad U, Henningsen MH, Gottschau B, Mathiesen O, et al. (2012) Effect of adductor-canal-blockade on established, severe post-operative pain after total knee arthroplasty: a randomized study. Acta Anaesthesiologica Scandinavica 56: 1013-1019.

22 Shah NA, Jain NP, Panchal KA (2015) Adductor Canal Blockade Following Total Knee Arthroplasty-Continuous or Single Shot Technique? Role in Postoperative Analgesia, Ambulation Ability and Early Functional Recovery: A Randomized Controlled Trial. J Arthroplasty 30: 1476-1481.

23 Ganapathy S, Wasserman RA, Watson JT, Bennett J, Armstrong KP, et al. (1999) Modified Continuous Femoral Three-in-One Block for Postoperative Pain after Total Knee Arthroplasty. Anaesthesia \& Analgesia 89: 1197-1202.

24 Kadic L, Boonstra MC, De Waal Malefijt MC, Lako SJ, Van Egmond $\mathrm{J}$, et al. (2009) Continuous femoral nerve block after total knee arthroplasty. Acta Anaesthesiologica Scandinavica 53: 914-920.

25 Shum CF, Lo NN, Yeo SJ, Yang KY, Chong HC, et al. (2009) Continuous femoral nerve block in total knee arthroplasty: immediate and twoyear outcomes. The Journal of arthroplasty 24: 204-209.

26 Seet E, Leong WL, Yeo AS, Fook-Chong S (2006) Effectiveness of 3-in1 continuous femoral block of differing concentrations compared to patient-controlled intravenous morphine for post total knee arthroplasty analgesia and knee rehabilitation. Anaesthesia and intensive care 34: 25 .

27 Salinas FV, Liu SS, Mulroy MF (2006) The effect of single-injection femoral nerve block versus continuous femoral nerve block after total knee arthroplasty on hospital length of stay and long-term functional recovery within an established clinical pathway. Anaesthesia \& Analgesia 102: 1234-1239.

28 Ilfeld BM, Le LT, Meyer RS, Mariano ER, Vandenborne K, et al. (2008) Ambulatory continuous femoral nerve blocks decrease time to discharge readiness after tricompartment total knee arthroplasty: a randomized, triple-masked, placebo-controlled study. Anaesthesiology 108: 703-713.

29 Jenstrup MT, Jaeger P, Lund J, Fomsgaard JS, Bache S, et al. (2012) Effects of Adductor-Canal-Blockade on pain and ambulation after total knee arthroplasty: a randomized study. Acta anaesthesiologica Scandinavica 56: 357-364.

30 Thapa D, Ahuja V, Verma P, Gombar S, Gupta R, et al. (2016) Postoperative analgesia using intermittent vs. continuous adductor canal block technique: a randomized controlled trial. Acta Anaesthesiologica Scandinavica. 